\title{
Magnetic structure of the magnetoelectric material $\mathrm{Ca}_{2} \mathrm{CoSi}_{2} \mathrm{O}_{7}$
}

\author{
A. Sazonov, ${ }^{1,}$ V. Hutanu, ${ }^{1}$ M. Meven, ${ }^{1}$ G. Roth, ${ }^{2}$ H. Murakawa, ${ }^{3,4}$ Y. Tokura, ${ }^{5,6}$ V. K. Guduru, ${ }^{7}$ L. C. J. M. Peters, ${ }^{7}$ U. Zeitler, ${ }^{7}$ \\ L. F. Kiss ${ }^{8}$ D. Szaller, ${ }^{9}$ B. Náfrádi, ${ }^{10}$ and I. Kézsmárki ${ }^{9}$ \\ ${ }^{1}$ Institute of Crystallography, RWTH Aachen University and Jülich Centre for Neutron Science (JCNS) at Heinz, Maier-Leibnitz, Zentrum \\ (MLZ), 85747 Garching, Germany \\ ${ }^{2}$ Institute of Crystallography, RWTH Aachen University, 52056 Aachen, Germany \\ ${ }^{3}$ Multiferroics Project, ERATO, Japan Science and Technology Agency (JST), University of Tokyo, Tokyo 113-8656, Japan \\ ${ }^{4}$ Department of Physics, Osaka University, 560-0043 Osaka, Japan \\ ${ }^{5}$ Department of Applied Physics and Quantum Phase Electronics Center (QPEC), University of Tokyo, Tokyo 113-8656, Japan \\ ${ }^{6}$ RIKEN Center for Emergent Matter Science (CEMS), Wako 351-0198, Japan \\ ${ }^{7}$ High Field Magnet Laboratory (HFML-EMFL) and Institute for Molecules and Materials, Radboud University, 6525 ED Nijmegen, \\ The Netherlands \\ ${ }^{8}$ Institute for Solid State Physics and Optics, Wigner Research Centre for Physics, Hungarian Academy of Sciences, 1525 Budapest, Hungary \\ ${ }^{9}$ Department of Physics, Budapest University and Economics of Technology and MTA-BME Lendület Magneto-optical Spectroscopy Research \\ Group, 1111 Budapest, Hungary \\ ${ }^{10}$ École Polytechnique Fédérale de Lausanne, Laboratory of Nanostructures \\ and Novel Electronic Materials, 1015 Lausanne, Switzerland \\ (Received 11 January 2017; published 22 May 2017)
}

\begin{abstract}
Detailed investigation of $\mathrm{Ca}_{2} \mathrm{CoSi}_{2} \mathrm{O}_{7}$ was performed in its low-temperature magnetoelectric state combining neutron diffraction with magnetization measurements on single crystals. The crystal and magnetic structures well below the antiferromagnetic transition temperature of $T_{\mathrm{N}} \approx 5.7 \mathrm{~K}$ were determined using neutron diffraction. Neutron diffraction data imply no structural phase transition from $10 \mathrm{~K}$ down to $2.5 \mathrm{~K}$ and are well described within the orthorhombic space group $P 2_{1} 2_{1} 2$ with a $3 \times 3 \times 1$ supercell compared with the high-temperature unmodulated state (tetragonal space group $P \overline{4} 2_{1} m$ ). We found that in zero magnetic field the magnetic space group is $P 2_{1} 2_{1}^{\prime} 2^{\prime}$ with antiferromagnetic order along the [100] or [010] axes for two types of $90^{\circ}$ twin domains, while neighboring spins along the [001] axis are ordered ferromagnetically. A noncollinear spin arrangement due to small canting within the $a b$ plane is allowed by symmetry and leads to the existence of the tiny spontaneous magnetization below $T_{\mathrm{N}}$. The ordered moment with a magnitude of about $2.8 \mu_{\mathrm{B}} / \mathrm{Co}^{2+}$ at $2.5 \mathrm{~K}$ lies in the $a b$ plane. Distinct differences between the magnetic structure of $\mathrm{Ca}_{2} \mathrm{CoSi}_{2} \mathrm{O}_{7}$ as compared to those of $\mathrm{Ba}_{2} \mathrm{CoGe}_{2} \mathrm{O}_{7}$ and $\mathrm{Sr}_{2} \mathrm{CoSi}_{2} \mathrm{O}_{7}$ are discussed.
\end{abstract}

DOI: 10.1103/PhysRevB.95.174431

\section{INTRODUCTION}

Recently, several members of the melilite family, such as $\mathrm{Ca}_{2} \mathrm{CoSi}_{2} \mathrm{O}_{7}, \mathrm{Sr}_{2} \mathrm{CoSi}_{2} \mathrm{O}_{7}, \mathrm{Ba}_{2} \mathrm{MnGe}_{2} \mathrm{O}_{7}$ and $\mathrm{Ba}_{2} \mathrm{CoGe}_{2} \mathrm{O}_{7}$, have been found to exhibit static as well as dynamic magnetoelectric effects. The dielectric properties of $\mathrm{Ca}_{2} \mathrm{CoSi}_{2} \mathrm{O}_{7}$ and its sister compounds have been studied in the magnetically ordered state [1-6]. In $\mathrm{Ca}_{2} \mathrm{CoSi}_{2} \mathrm{O}_{7}$ a remarkably large magnetocapacitance effect, reaching the value of $\Delta \varepsilon / \varepsilon=13 \%$, was observed in $B=8 \mathrm{~T}$ at $T=5.1 \mathrm{~K}$. Moreover, it was demonstrated $[3,7,8]$ via polarized terahertz spectroscopy studies on multiferroic $\mathrm{Ca}_{2} \mathrm{CoSi}_{2} \mathrm{O}_{7}, \mathrm{Sr}_{2} \mathrm{CoSi}_{2} \mathrm{O}_{7}$, and $\mathrm{Ba}_{2} \mathrm{CoGe}_{2} \mathrm{O}_{7}$ that their magnetoelectric spin excitations exhibit quadrochroism: They have different colors for all four combinations of two propagation directions (forward or backward) and two orthogonal polarizations of a light beam.

This compound family is usually referred to as a representative class of type-II multiferroics in the literature, where the magnetically induced polarization originates from spindependent $p$ - $d$ hybridization between the transition metal ions and their ligands. However, strictly speaking these compounds are magnetoelectric but not multiferroic, since the development of magnetic order is accompanied with ferroelectric

\footnotetext{
*mail@ sazonov.org; http://sazonov.org
}

polarization in finite magnetic fields and in zero field only spin-induced antiferroelectricity is present in these materials.

The main features of the magnetoelectric behavior of $\mathrm{Ba}_{2} \mathrm{CoGe}_{2} \mathrm{O}_{7}$ were predicted [9] by symmetry considerations without appealing to any specific atomic mechanism. However, for many melilite systems, specific structural information is still unavailable.

Due to the lack of the low-temperature structural details, a general noncentrosymmetric tetragonal structure of melilites $A_{2} B_{T O} \mathrm{O}_{7}$ with space group (SG) $P \overline{4} 2_{1} m$ is often used $[3,4,10]$. In $\mathrm{Ba}_{2} \mathrm{CoGe}_{2} \mathrm{O}_{7}$, the room temperature crystal structure is indeed tetragonal $P \overline{4} 2{ }_{1} m$ with $a=b \approx 8.39 \AA$ and $c \approx 5.56 \AA$ (Ref. [11]), with possible symmetry lowering to orthorhombic $C m m 2$ at low temperatures $[11,12]$. The room temperature crystal structure of $\mathrm{Sr}_{2} \mathrm{CoSi}_{2} \mathrm{O}_{7}$ is also tetragonal $P \overline{4} 2_{1} m$ with $a=b \approx 8.03 \AA$ and $c \approx 5.16 \AA$ (Ref. [13]). However, $\mathrm{Ca}_{2} \mathrm{CoSi}_{2} \mathrm{O}_{7}$ for example undergoes a series of structural phase transitions from $600 \mathrm{~K}$ down to $30 \mathrm{~K}$ [14-17]. The first one corresponds to a transition from the normal (unmodulated) phase to the incommensurately modulated one (N-IC) at $T_{\mathrm{N}-\mathrm{IC}} \approx$ $480-500 \mathrm{~K}$. The second transition is an incommensurate-tocommensurate structural change (IC-C), where the published $T_{\text {IC-C }}$ varies in a broad temperature range between 160 and $270 \mathrm{~K}[15,18,19]$. Accordingly, in the high temperature unmodulated state (above $T_{\mathrm{N}-\mathrm{IC}}$ ) the $\mathrm{SG}$ of $\mathrm{Ca}_{2} \mathrm{CoSi}_{2} \mathrm{O}_{7}$ is $P \overline{4} 2_{1} m$ with lattice parameters $a=b \approx 7.86 \AA$ and $c \approx$ 
$5.03 \AA$ (Ref. [14]). In the intermediate temperature range (between $T_{\mathrm{IC}-\mathrm{C}}$ and $T_{\mathrm{N}-\mathrm{IC}}$ ), an incommensurately modulated phase develops with two propagation vectors $\mathbf{k}_{1}=q\left(\mathbf{a}^{*}+\mathbf{b}^{*}\right)$ and $\mathbf{k}_{2}=q\left(-\mathbf{a}^{*}+\mathbf{b}^{*}\right)$, where $q$ increases with decreasing temperature from about 0.29 up to $1 / 3$ (Ref. [15]). At low temperatures (below $T_{\text {IC-C }}$ ), a commensurately modulated lock-in structure appears with a $3 \times 3 \times 1$ supercell and lattice parameters $a \approx b \approx 23.51 \AA$ and $c \approx 5.03 \AA$ (Refs. [15,16]).

It is clear that precise information for the atomic positions as well as about the spin order (crystal and magnetic structures) is essential to unravel the complex physics behind the magnetoelectric behavior of the melilite compounds. Recently, detailed structural investigation of $\mathrm{Ca}_{2} \mathrm{CoSi}_{2} \mathrm{O}_{7}$ by means of neutron diffraction were performed [17] at $10 \mathrm{~K}$, just above $T_{\mathrm{N}} \approx 5.7 \mathrm{~K}$. The precise structural parameters within the twinned orthorhombic crystal with space group $P 22_{1} 2{ }_{1}$ and $3 \times 3 \times 1$ supercell were deduced and compared with the $\mathrm{X}$-ray diffraction results in the low-temperature commensurate phase (130 and $170 \mathrm{~K})$, as well as in the high-temperature unmodulated phase $(500 \mathrm{~K})$.

The magnetic structure of $\mathrm{Ca}_{2} \mathrm{CoSi}_{2} \mathrm{O}_{7}$ is considered $[3,4,10]$ to be similar to that of $\mathrm{Ba}_{2} \mathrm{CoGe}_{2} \mathrm{O}_{7}$, where the magnetic space group is $C m^{\prime} m 2^{\prime}$ with antiferromagnetic order parallel to the [100] axis of this orthorhombic setting (or [110] in the high-temperature tetragonal SG $P \overline{4} 2_{1} m$ ). However, the different parent symmetries of $\mathrm{Ca}_{2} \mathrm{CoSi}_{2} \mathrm{O}_{7}\left(\mathrm{SG} P 2_{1} 2_{1} 2\right)$ and $\mathrm{Ba}_{2} \mathrm{CoGe}_{2} \mathrm{O}_{7}\left(\mathrm{SG} P \overline{4} 2_{1} m\right)$ in the paramagnetic state can easily lead to different ground-state magnetic structures. More specifically, the single-ion anisotropy-arising from the axial deformation of the $\mathrm{CoO}_{4}$ tetrahedra in the tetragonal structure-is a key parameter for determining the nature of the magnetic ground state and the low-lying excited states. $\mathrm{Ba}_{2} \mathrm{CoGe}_{2} \mathrm{O}_{7}$, where single-ion anisotropy acting on the $S=3 / 2$ spins of $\mathrm{Co}^{2+}$ ions is even stronger than the first neighbor exchange interactions, is a Néel-type squarelattice antiferromagnet with strong easy-plane character. As a dynamic manifestation of the strong on-site anisotropy, spin stretching modes have been observed in the magnon excitation spectrum of this compound [20,21]. However, the difference between the lattice parameters of $\mathrm{Ba}_{2} \mathrm{CoGe}_{2} \mathrm{O}_{7}\left(a_{\text {tet }} \approx 8.35 \AA\right.$, $\left.c_{\text {tet }} \approx 5.50 \AA\right), \mathrm{Sr}_{2} \mathrm{CoSi}_{2} \mathrm{O}_{7}\left(a_{\text {tet }} \approx 8.03 \AA, c_{\text {tet }} \approx 5.16 \AA\right)$ and $\mathrm{Ca}_{2} \mathrm{CoSi}_{2} \mathrm{O}_{7}\left(a_{\text {tet }} \approx 7.86 \AA, c_{\text {tet }} \approx 5.03 \AA\right)$ may result in different magnetic ground states for these compounds. Based on the distortion of the $\mathrm{CoO}_{4}$ tetrahedra in the various cases, we expect different strengths of the single-ion anisotropies.

In order to fill this gap of magnetic structure information on $\mathrm{Ca}_{2} \mathrm{CoSi}_{2} \mathrm{O}_{7}\left(T_{\mathrm{N}} \approx 5.7 \mathrm{~K}\right)$ and to provide reliable data for further experimental and theoretical research, we performed single-crystal neutron diffraction experiments as well as bulk magnetization measurements at temperatures between 1.7 and $10 \mathrm{~K}$ and fields up to $32 \mathrm{~T}$. The observed magnetic properties are compared with those of $\mathrm{Ba}_{2} \mathrm{CoGe}_{2} \mathrm{O}_{7}\left(T_{\mathrm{N}} \approx 6.7 \mathrm{~K}\right)$ and $\mathrm{Sr}_{2} \mathrm{CoSi}_{2} \mathrm{O}_{7}\left(T_{\mathrm{N}} \approx 7.0 \mathrm{~K}\right)$.

\section{EXPERIMENT}

Single crystals of $\mathrm{Ca}_{2} \mathrm{CoSi}_{2} \mathrm{O}_{7}$ were grown by the floatingzone technique and characterized in our previous studies (see Ref. [17] and references therein). The cylindrical sample used for neutron scattering experiments was approximately $4 \mathrm{~mm}$ high with about the same diameter.
Unpolarized single-crystal neutron diffraction studies were performed on the four-circle diffractometer HEiDi (Ref. [22]) at the hot-neutron source of the FRM II reactor, Heinz Maier-Leibnitz Zentrum (MLZ), Germany. A wavelength $\lambda=1.169 \AA$ was obtained from a $\mathrm{Ge}(311)$ monochromator with a high flux density of about $1.2 \times 10^{7}$ neutrons s${ }^{-1} \mathrm{~cm}^{-1}$ with an Er filter to suppress the $\lambda / 3$ contamination. For low-temperature experiments, a closed-cycle $\mathrm{He}$ cryostat was mounted in the Eulerian cradle of the diffractometer. The sample was wrapped in $\mathrm{Al}$ foil in order to ensure temperature homogeneity. The temperature was measured and controlled by a diode sensor near the heater position. The sample temperature was independently monitored by a second thermometer placed close to the sample position. A temperature stability of $\pm 0.1 \mathrm{~K}$ was achieved by this method. Temperature dependences of the selected Bragg reflections were measured in the range of 2.2 to $10 \mathrm{~K}$ during the cooling process. Full data collection was done at $2.5 \mathrm{~K}$.

An additional neutron-scattering experiment was performed on the cold triple-axis spectrometer TASP at the Swiss Spallation Neutron Source (SINQ), Paul Scherrer Institut (PSI), Switzerland. Neutrons with a fixed final neutron energy of $14.7 \mathrm{meV}$ were used. Vertically focused pyrolitic graphite monochromators and horizontally focused analyzers, as well as Be filters positioned after the sample, were utilized. The sample was mounted with the [001] axis vertical, making momentum transfer in the $(h k 0)$ reciprocal-space plane accessible for measurements. The sample environment was the standard "ILL Orange" He-flow cryostat.

The experimental (observed) structure factors of the measured Bragg reflections were obtained with the DAVINCI program [23] using the Lehmann-Larsen method for peak location [24]. The crystal and magnetic structure parameters of $\mathrm{Ca}_{2} \mathrm{CoSi}_{2} \mathrm{O}_{7}$ were refined using the JANA2006 program [25].

The field dependence of the magnetization up to $32 \mathrm{~T}$ was measured using a vibrating sample magnetometer in a Bitter magnet, High Field Magnet Laboratory, Nijmegen. The experiments were performed at $T=2,4$, and $6 \mathrm{~K}$ in fields parallel to the [110], [100], and [001] crystallographic directions. The absolute magnetic moment was confirmed by magnetization measurements performed in the 0-5 $\mathrm{T}$ field range using a Quantum Design MPMS-5S superconducting quantum interference device (SQUID) magnetometer in DC fields.

The angular dependences of the magnetization were measured in a $7 \mathrm{~T}$ superconducting magnet equipped with a DC magnetometer. During the experiment, the sample was rotated around the [001] axis, perpendicular to the magnetic field, and the magnetization parallel to the applied field was detected. The initial direction of the single crystal was set under an optical microscope with about $5^{\circ}$ precision. The direction was changed by pulling the string which was tied to the rotation axis of the sample stage. This procedure allowed a control of the sample orientation by about $1^{\circ}$.

\section{RESULTS AND DISCUSSION}

\section{A. Magnetic structure models}

The low-temperature commensurately modulated lock-in state of $\mathrm{Ca}_{2} \mathrm{CoSi}_{2} \mathrm{O}_{7}$ at $10 \mathrm{~K}$ (Ref. [17]) is described by the orthorhombic space group $P 2{ }_{1} 2{ }_{1} 2$ with a $3 \times 3 \times 1$ supercell 


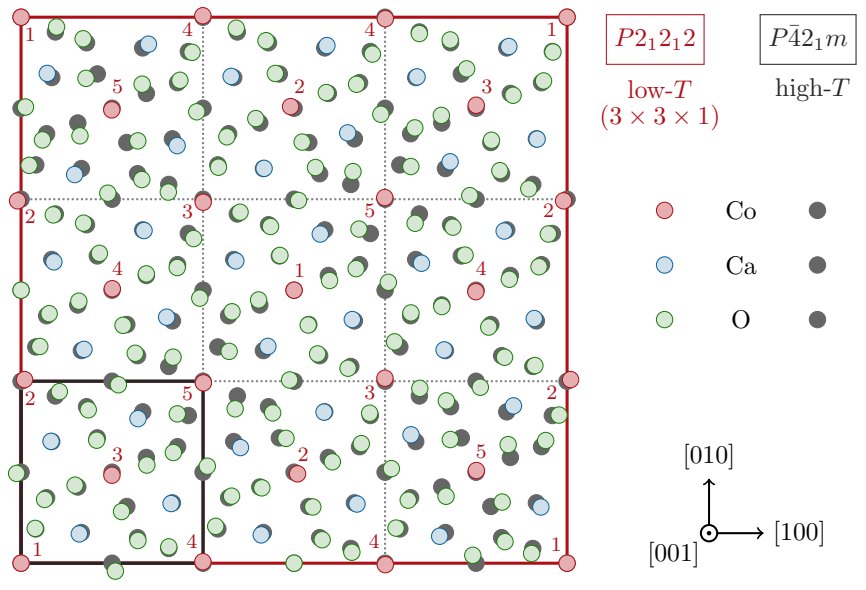

FIG. 1. Comparison of the atomic shift between the lowtemperature commensurately modulated lock-in state (SG $P 2_{1} 2_{1} 2$ ) and the high-temperature unmodulated phase (SG $P \overline{4} 2_{1} m$ ), according to our neutron diffraction data at $2.5 \mathrm{~K}$ and $\mathrm{x}$-ray diffraction at about $500 \mathrm{~K}$ (Ref. [14]), respectively. The small gray square corresponds to the unit cell of the high-temperature unmodulated phase with SG $P \overline{4} 2{ }_{1} m$ and the large red one to the $3 \times 3 \times 1$ supercell of SG $P 2{ }_{1} 2_{1} 2$. Five inequivalent cobalt sites are numbered. Silicon atoms are not shown for simplicity; Their shifts are small and comparable with those of cobalt.

compared to the high-temperature unmodulated phase with space group $P \overline{4} 2_{1} m$. Below $T_{\mathrm{N}} \approx 5.7 \mathrm{~K}$, an additional magnetic contribution to the Bragg reflection intensities appears corresponding to the commensurate magnetic order with propagation vector $\boldsymbol{k}=\mathbf{0}$. Thus, the unit cells of the magnetic and paramagnetic structures are found to be identical. No indication of a symmetry change was found from $10 \mathrm{~K}$ down to $2.2 \mathrm{~K}$ according to the neutron diffraction data. Thus, within the experimental precision the crystal structure remains $P 2{ }_{1} 2{ }_{1} 2$ also in the magnetic phase.

The high-temperature Wyckoff position (WP) $2 a(0,0,0)$ of the magnetic $\mathrm{Co}^{2+}$ ions splits in the low-temperature $3 \times 3 \times 1$ supercell into five positions: one special $2 a(0,0, z)$ labeled hereafter as Co1 and four general $4 c(x, y, z)$ labeled as Co2-5. The shift of Co is found to be relatively small and the symmetry lowering is mostly governed by the positional change of the part of the nonmagnetic $\mathrm{Ca}$ and $\mathrm{O}$ ions (Fig. 1). The shifts of the $\mathrm{Si}$ atoms are also weak and they are not shown in the figure for simplicity. The nonmagnetic atoms are irrelevant for magnetic diffraction, but they can be important to identify the magnetic symmetry [26].

In order to solve the magnetic structure of $\mathrm{Ca}_{2} \mathrm{CoSi}_{2} \mathrm{O}_{7}$ we used the concept of Shubnikov groups (magnetic space groups, MSGs), which is very useful in the case of second-order phase transitions for enumerating the possible magnetic structures compatible with the crystal symmetry. This approach implies specific symmetry-deduced constraints on the magnetic moments. That is, the magnetic moments of symmetry equivalent atoms are related via the magnetic symmetry operations. It allows one to reduce the number of refined parameters and to average the symmetry equivalent reflections. It was shown that the use of MSGs significantly facilitates the interpretation of the results (see, e.g., Ref. [26] and references therein).

The nonmagnetic parent space group of $\mathrm{Ca}_{2} \mathrm{CoSi}_{2} \mathrm{O}_{7}$ is $P 22_{1} 2$ and its corresponding gray group is $P 2{ }_{1} 2{ }_{1} 21^{\prime}$, which in addition includes the time reversal operation. The symmetry of a magnetically ordered phase is described by a subgroup of this parent group. Figure 2 shows the $k$-maximal subgroups for $P 2{ }_{1} 2{ }_{1} 21^{\prime}$ with the magnetic propagation vector $\boldsymbol{k}=\mathbf{0}$. Only four distinct types of magnetic ordering of $k$-maximal symmetry are possible in $\mathrm{Ca}_{2} \mathrm{CoSi}_{2} \mathrm{O}_{7}$ : model $M 1$ (MSG $P 2_{1} 2_{1} 2$ ), model $M 2$ (MSG $P 2_{1}^{\prime} 2_{1}^{\prime} 2$ ), model $M 3$ (MSG $P 2_{1} 2_{1}^{\prime} 2^{\prime}$ ), and model $M 4$ (MSG $P 2_{1} 2_{1}^{\prime} 2^{\prime}$ ). The differences between the models manifest themselves in the distinct magnetic modes which can be presented as the antiferromagnetic (AF) and ferromagnetic $(\mathrm{F})$ components along the crystallographic axes $a, b$, and $c$. F alignment is completely forbidden for the $M 1$ case, while it is allowed in $M 2$ (along $c$ axis), M3 (along $a$ axis), and $M 4$ (along $b$ axis). The full set of possible components is given in Fig. 2. The combination of the modes varies for two types of cobalt sites of different symmetry: Co1, WP $2 a(0,0, z)$ and Co2-5, WP $4 c(x, y, z)$. As can be

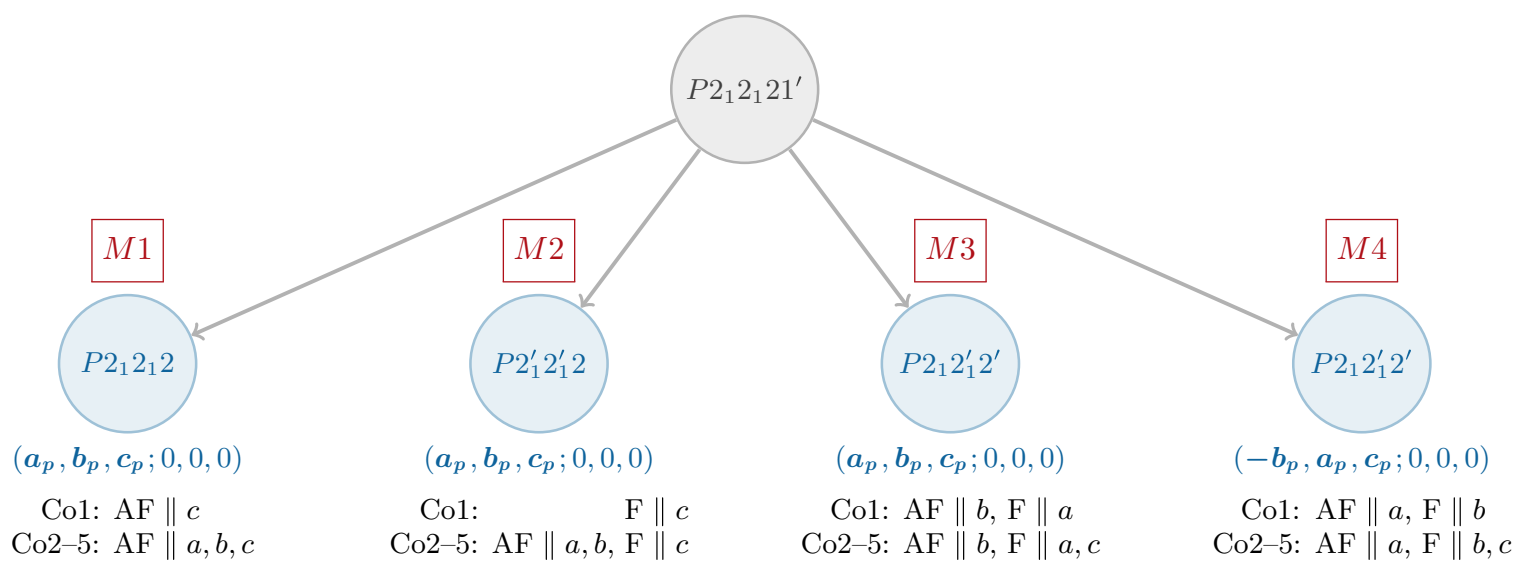

FIG. 2. The possible $k$-maximal symmetries for a magnetic ordering of Co with propagation vector $\boldsymbol{k}=\mathbf{0}$ on a paramagnetic phase with space group $P 2{ }_{1} 2{ }_{1} 2$ and its corresponding gray group $P 2{ }_{1} 2{ }_{1} 21^{\prime}$. The magnetic space group label is shown together with the transformation from the parent $P 2{ }_{1} 2{ }_{1} 21^{\prime}$ unit cell basis $\left\{\boldsymbol{a}_{p}, \boldsymbol{b}_{p}, \boldsymbol{c}_{p}\right\}$ to its standard setting. The possible antiferromagnetic (AF) and ferromagnetic (F) components along the crystallographic directions $a, b$, and $c$ are given at the bottom separately for the Co1 and Co2-5 sites. 
seen from the diagram, the $M 3$ and $M 4$ models differ only by the interchange of the $a$ and $b$ axes.

\section{B. Magnetic structure refinement at $2.5 \mathrm{~K}$}

All four symmetry-allowed magnetic structure models mentioned above were used in the refinement process to compare the experimental data with calculations. The index of all the MSGs of $\mathrm{Ca}_{2} \mathrm{CoSi}_{2} \mathrm{O}_{7}$ with respect to the parent symmetry $P 2{ }_{1} 2{ }_{1} 21^{\prime}$ is two. Thus, only a trivial magnetic twin with all spins reversed is possible, having no consequence on the diffraction data. However, the crystal structure of $\mathrm{Ca}_{2} \mathrm{CoSi}_{2} \mathrm{O}_{7}$ at low temperatures was found to consist already of two individuals of the same space group symmetry which are twinned with respect to a diagonal mirror plane $m_{x y}\left(90^{\circ}\right.$ twin domains) [15-17]. The twin fraction ${ }^{1}$ from the present data at $2.5 \mathrm{~K}$ is refined to be $0.52(1)$, which is the same as at $10 \mathrm{~K}$ (Ref. [17]) and very close to a perfect twin value of 0.5 . If the structural twin domains are equally populated, the difference between the magnetic models $M 3$ and $M 4$ is hidden. As a result, we combine these two magnetic structures into a single twinned model $M 3 / M 4$.

A total of 4790 reflections with $\sin \theta / \lambda \lesssim 0.7 \AA^{-1}$ were measured at $2.5 \mathrm{~K}$, and 2779 unique reflections were obtained by averaging equivalents ${ }^{2}\left(R_{\text {int }}=0.042\right)$.

In the first step of the refinement, all the structural parameters for $\mathrm{Ca}_{2} \mathrm{CoSi}_{2} \mathrm{O}_{7}$ were fixed according to the results of the neutron diffraction measurements at $10 \mathrm{~K}$ (Ref. [17]) and only the magnetic structure components were refined. Finally, the nuclear and magnetic structures were refined simultaneously.

The main contribution to most of the Bragg peaks is caused by nuclear scattering. Therefore, a direct comparison of the whole set of few thousand reflections is not demonstrative enough. In order to clearly illustrate the difference between the models, a few representative pure magnetic, e.g., (300)/(030), or mixed magnetic and nuclear, e.g., (301)/(031), reflections were selected for comparison (Fig. 3). We note that the whole data set was used in the refinement process, taking into account $90^{\circ}$ twinning, and the final fit in the full $M 3 / M 4$ model (14 refined parameters) is presented in Fig. 3(f). For comparison, the fit quality of the model without magnetic contribution (nuclear only model) is also given in Fig. 3(a). As can be seen from the figure, both model $M 1$ [Fig. 3(b)] and M2 [Fig. 3(c)] are not able to fully reproduce the intensities of the magnetic reflections and only the twinned model $M 3 / M 4$ [Fig. 3(d)] fits the experimental data well.

The large number of the refined magnetic structure components (14 parameters) as well as the weak magnetic contributions on top of nuclear ones for the major part of the reflections lead to quite large uncertainties in the refined parameters (see Table I). The small $\mathrm{F}$ components in the $a b$ plane and along the $c$ axis are found to be comparable with their standard

\footnotetext{
${ }^{1}$ The twin fraction represents the fractional volume of the crystal that the second domain occupies.

${ }^{2}$ The accuracy of the averaged intensities is estimated from the spread of the individual measurements of equivalent reflections by $R_{\text {int }}=\sum\left|F_{\text {obs }}^{2}-\left\langle F_{\text {obs }}^{2}\right\rangle\right| / \sum F_{\mathrm{obs}}^{2}$.
}

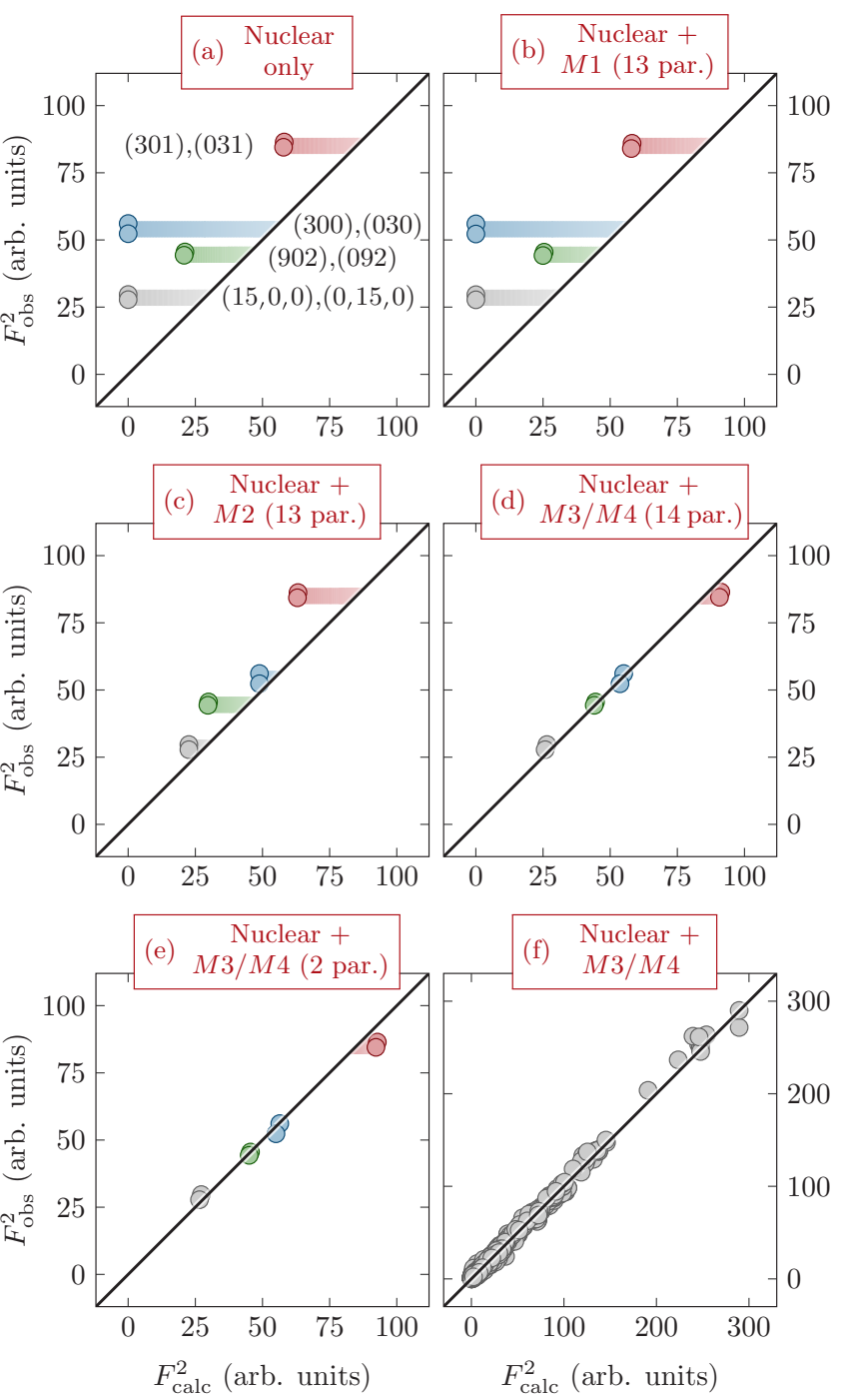

FIG. 3. Quality of the neutron diffraction data refinement at $2.5 \mathrm{~K}$. The observed structure factors squared $\left(F_{\mathrm{obs}}^{2}\right)$ are plotted against the calculated ones $\left(F_{\text {calc }}^{2}\right)$. Eight representative Bragg reflections were selected to illustrate the fit quality considering (a) the pure nuclear structure or possible magnetic models (b) $M 1$, (c) $M 2$, and (d) $M 3 / M 4$. The simplified (e) $M 3 / M 4$ model with only 2 refined parameters is also presented for comparison. A fit of (f) the full data set is given in the unrestricted $M 3 / M 4$ model (14 refined parameters) to evaluate the quality of the final simultaneous refinement of $\mathrm{Ca}_{2} \mathrm{CoSi}_{2} \mathrm{O}_{7}$ crystal and magnetic structures.

deviations and thus can be approximated to zero. Therefore, we performed an additional refinement in the simplified magnetic model which assumes that all the cobalt atoms have (1) equal in-plane $\mathrm{AF}$ moments, (2) equal in-plane $\mathrm{F}$ components, and (3) zero out-of-plane F components (see Table I). Strictly speaking, these simplifications are not symmetry protected, but allow one to describe the experimental data with the same quality [see Fig. 3(e)] with only two refined parameters, i.e., the $\mathrm{AF} \|[100]$ and $\mathrm{F} \|[010]$ components of the cobalt magnetic moment. The good agreement with these constraints being implemented can be associated with the relatively small shifts of the Co ions from their high-symmetry position in the 
TABLE I. Magnetic moment components $\left(\mu_{\mathrm{B}}\right)$ of the symmetryindependent $\mathrm{Co}$ atoms in the twinned $M 3 / M 4$ and its simplified magnetic structures models, as refined from neutron diffraction data at $2.5 \mathrm{~K}$. Cobalt atomic coordinates are given in Table II.

\begin{tabular}{lccccc}
\hline \hline Atom & WP & $M_{x}$ & $M_{y}$ & $M_{z}$ & $|M|$ \\
\hline \multicolumn{7}{l}{ Full model $M 3 / M 4(14$ parameters) } \\
Co1 & $2 e$ & $2.98(31)$ & $0.21(27)$ & 0 & $2.98(32)$ \\
Co2 & $4 c$ & $2.62(18)$ & $0.04(19)$ & $0.20(41)$ & $2.63(19)$ \\
Co3 & $4 c$ & $-2.76(14)$ & $-0.27(41)$ & $0.16(44)$ & $2.78(17)$ \\
Co4 & $4 c$ & $2.94(22)$ & $-0.12(24)$ & $0.07(33)$ & $2.95(22)$ \\
Co5 & $4 c$ & $2.74(12)$ & $-0.29(44)$ & $-0.09(48)$ & $2.76(13)$ \\
Simplified model $M 3 / M 4(2$ parameters $)$ & & \\
Co1 & $2 e$ & $2.79(1)$ & $-0.07(6)$ & 0 & $2.80(1)$ \\
Co2 & $4 c$ & $2.79(1)$ & $-0.07(6)$ & 0 & $2.80(1)$ \\
Co3 & $4 c$ & $-2.79(1)$ & $-0.07(6)$ & 0 & $2.80(1)$ \\
Co4 & $4 c$ & $2.79(1)$ & $-0.07(6)$ & 0 & $2.80(1)$ \\
Co5 & $4 c$ & $2.79(1)$ & $-0.07(6)$ & 0 & $2.80(1)$ \\
\hline \hline
\end{tabular}

high-temperature unmodulated state with SG $P \overline{4} 2_{1} m$ (Fig. 1). This is also in agreement with the observation that the strongest magnetic contributions appear in Bragg reflections with $h=$ $3 n$ and $k=3 n \quad(n \in \mathbb{Z})$. The simplified magnetic model $M 3 / M 4$ would correspond to MSG $P 2{ }_{1} 2_{1}^{\prime} 2^{\prime}$ derived from the parent gray group $P \overline{4} 2_{1} m 1^{\prime}$ with the normal $1 \times 1 \times 1$ unit cell and $\boldsymbol{k}=0$. However, the choice of MSG depends in general not only on the magnetic atoms but also on the actual positions of the nonmagnetic ones. Therefore, despite their irrelevance in magnetic diffraction, nonmagnetic atoms play an important role in the symmetry of a magnetic phase. Those atoms should be taken into account independently of the simplicity of the spin arrangement when identifying the magnetic symmetry [26]. Thus, only the full $M 3 / M 4$ model (MSG $P 2{ }_{1} 2_{1}^{\prime} 2^{\prime}, 3 \times 3 \times 1$ cell) agrees with the crystal structure at low temperatures.

Both twinned M3/M4 (14 parameters) and its simplified (2 parameters) magnetic structure models are shown and compared in Fig. 4. In both cases, the spin canting is allowed by the symmetry via the F components perpendicular to the direction of the primary AF ordering. However, the canting is found to be weak if not absent.

The average ordered magnetic moment in $\mathrm{Ca}_{2} \mathrm{CoSi}_{2} \mathrm{O}_{7}$ at $2.5 \mathrm{~K}$ as refined from neutron diffraction data is found to be about $2.8 \mu_{\mathrm{B}} / \mathrm{Co}$ (Table I), which is comparable with $2.81 \mu_{\mathrm{B}}$ observed for $\mathrm{Ba}_{2} \mathrm{CoGe}_{2} \mathrm{O}_{7}$ [12]. This value found for $\mathrm{Ca}_{2} \mathrm{CoSi}_{2} \mathrm{O}_{7}$ is lower than the high-field saturation moment which exceeds $3.3 \mu_{\mathrm{B}} /$ Co (Ref. [13]), most probably as a consequence of the single-ion anisotropy induced splitting between the $S_{z}= \pm \frac{1}{2}$ and $S_{z}=\frac{3}{2}$, similarly to $\mathrm{Ba}_{2} \mathrm{CoGe}_{2} \mathrm{O}_{7}$ [12]. Since the lowest energy $S_{z}= \pm \frac{1}{2}$ levels are separated from the higher lying $S_{z}= \pm \frac{3}{2}$ states, the spin length, i.e., the expectation value of the static ordered moment, is reduced from $3 \mu_{\mathrm{B}} / \mathrm{Co}$, the spin-only value expected without anisotropy. On the other hand, the fact that the saturation moment exceeds $3 \mu_{\mathrm{B}} / \mathrm{Co}$ indicates orbital contribution to the magnetization.

However, the magnetic structure of $\mathrm{Ca}_{2} \mathrm{CoSi}_{2} \mathrm{O}_{7}$ with primary AF ordering along [100] tet, determined by symmetry

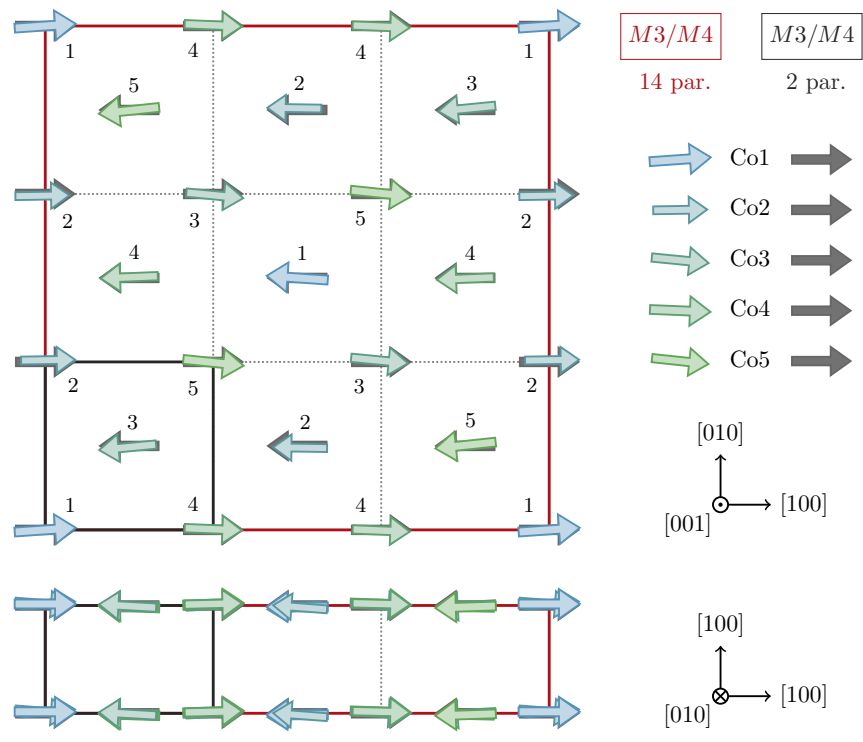

FIG. 4. Magnetic structure in the full $M 3 / M 4$ (14 parameters) and its simplified ( 2 parameters) models, according to our neutron diffraction data at $2.5 \mathrm{~K}$. The small unit cell of the high-temperature unmodulated phase with SG $P \overline{4} 2_{1} m$ is plotted in gray color and the large $3 \times 3 \times 1$ supercell of SG $P 22_{1} 2_{1} 2$ is shown in red. Five inequivalent cobalt sites are numbered (see also Fig. 1 for comparison).

analysis and neutron diffraction, differs from that proposed earlier for $\mathrm{Ca}_{2} \mathrm{CoSi}_{2} \mathrm{O}_{7}$ (Refs. [4,13]) and that found for $\mathrm{Ba}_{2} \mathrm{CoGe}_{2} \mathrm{O}_{7}$ (Ref. [12]), where the direction of the AF moment is [110 $]_{\text {tet }}$. Therefore, this should be taken into account for further theoretical and experimental investigations.

\section{Temperature evolution of magnetic structure}

In order to follow the temperature evolution of the magnetic structure of $\mathrm{Ca}_{2} \mathrm{CoSi}_{2} \mathrm{O}_{7}$, several intense magnetic and structural Bragg reflections were collected in the temperature range from 2.2 to $10 \mathrm{~K}$. Figure 5 (upper panel) shows the temperature dependences of the normalized integrated intensities of the magnetic Bragg reflection (300), as an example. These intensities decrease continuously with increasing temperature and become constant and close to zero above $T_{\mathrm{N}}$.

The integrated intensities $I$ of magnetic Bragg reflections measured with unpolarized neutrons follow the square of the magnetic order parameter. The data were fitted in the temperature range from $0.8 T_{\mathrm{N}}$ to $T_{\mathrm{N}}$, assuming a power law dependence, to the equation $[27,28]$

$$
I=I_{\mathrm{n}}+I_{0}\left(\frac{T_{\mathrm{N}}-T}{T_{\mathrm{N}}}\right)^{2 \beta},
$$

where $I_{\mathrm{n}}$ is the nuclear (structural) contribution to the intensity, $I_{0}$ is the magnetic intensity at $T=0$, and $\beta$ is the critical exponent.

The fit yields $\beta=0.20 \pm 0.04$ as critical exponent, which is close to the values found for $\mathrm{Ba}_{2} \mathrm{CoGe}_{2} \mathrm{O}_{7}$ (Ref. [12]) and other layered two-dimensional antiferromagnets [29-31]. This is in agreement with the layered crystal structure of $\mathrm{Ca}_{2} \mathrm{CoSi}_{2} \mathrm{O}_{7}$, where $\mathrm{CoO}_{4}$ and $\mathrm{Si}_{2} \mathrm{O}_{7}$ groups in the planes are separated 

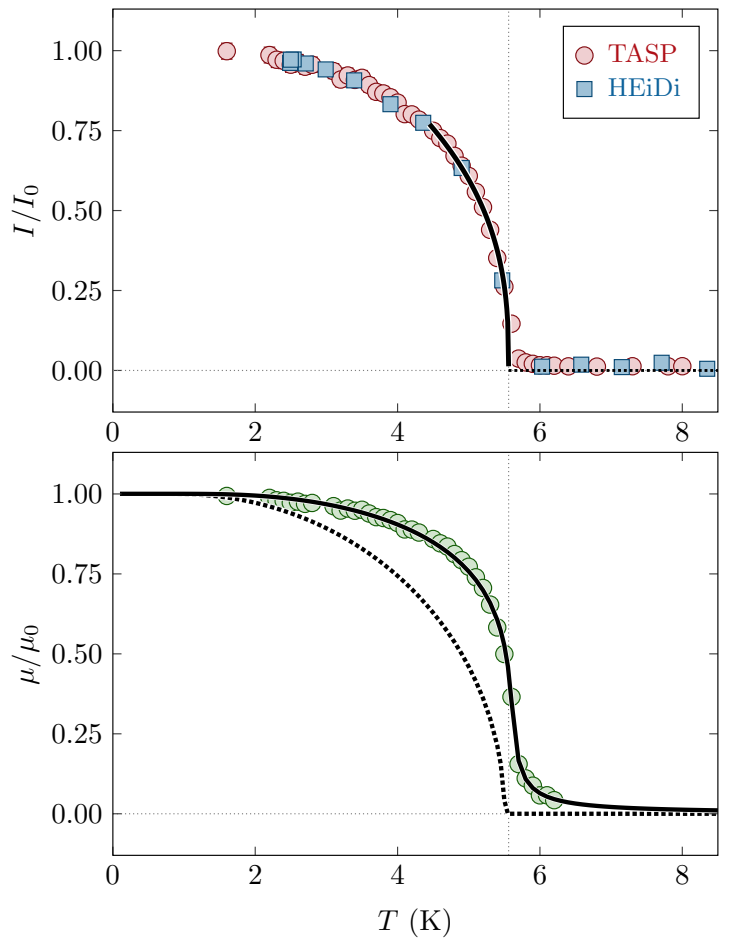

FIG. 5. Upper panel: Temperature dependences of the normalised integrated intensity of the magnetic Bragg reflection (300). Symbols correspond to the unpolarized single-crystal neutron diffraction data measured by two instruments: HEiDi and TASP. The solid line shows a fit to Eq. (1). Lower panel: Temperature dependence of the normalized Co magnetic moment. The experimental data from the single-crystal neutron diffraction measurements are shown by circles. The solid line is a result of a modified molecular field model [Eq. (3)]. The dashed line is shown to illustrate the deviation of $\mu(T)$ from the conventional molecular field model [Eq. (2)].

by interlayer $\mathrm{Ca}$ cations, and exchange interactions between cobalt spins on neighgoring layers are expected to be much weaker than intra-plane exchange couplings.

A very weak nuclear contribution above $T_{\mathrm{N}}$ is associated with the multiple diffraction or the Renninger effect (see, e.g., Refs. [32,33] and references therein), which was found in $\mathrm{Ca}_{2} \mathrm{CoSi}_{2} \mathrm{O}_{7}$ at $10 \mathrm{~K}$ (Ref. [17]) and $\mathrm{Ba}_{2} \mathrm{CoGe}_{2} \mathrm{O}_{7}$ at room temperature (Ref. [11]).

The lower panel of Fig. 5 shows the temperature dependence of the normalized Co magnetic moment. For a simple antiferromagnetic structure, the temperature dependence of the magnetic moment $\mu$ in the conventional molecular-field model can be expressed as

$$
\frac{\mu}{\mu_{0}}=B_{S}\left(\frac{3 S}{S+1} \frac{T_{\mathrm{N}}}{T} \frac{\mu}{\mu_{0}}\right),
$$

where $S$ is the spin of the system, $\mu_{0}$ is the magnetic moment at $T=0 \mathrm{~K}$, and $B_{S}$ is the Brillouin function.

This simple model with $S=3 / 2$ [high-spin (HS) state of $\mathrm{Co}^{2+}, t_{2 g}^{5} e_{g}^{2}$ ] fails to reproduce the experimental data, as shown by the dashed line in the lower panel of Fig. 5. While the molecular field theory predicts a sharp onset of the order parameter below $T_{\mathrm{N}}$, the experimental magnetic moment value starts to grow at higher temperatures above $T_{\mathrm{N}}$. Moreover, the
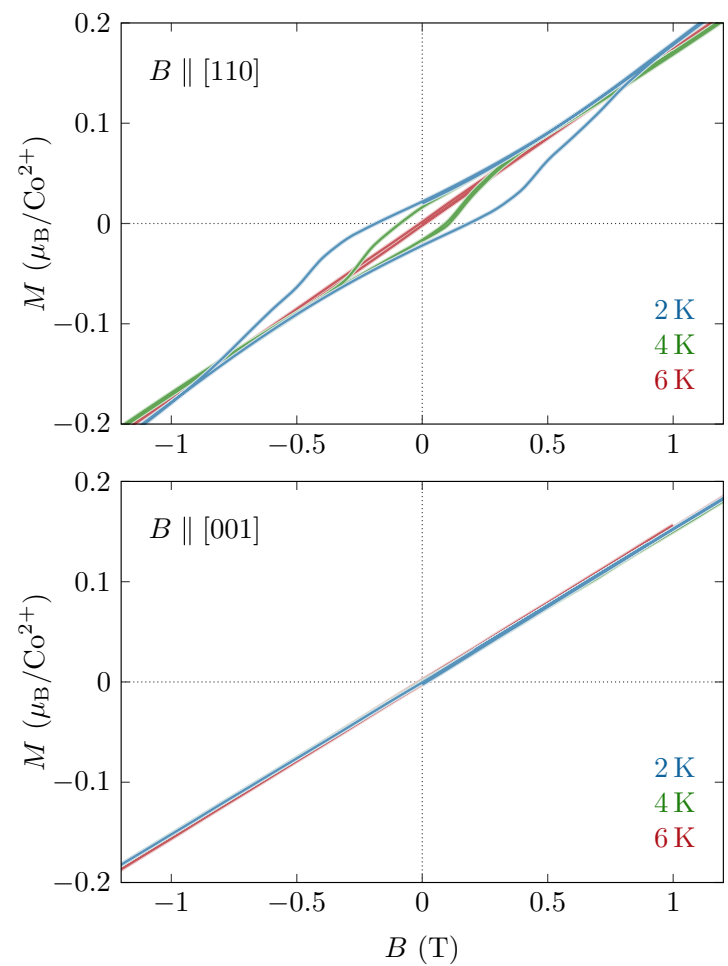

FIG. 6. Field dependences of the magnetization $M$ at $2 \mathrm{~K}$ (blue), $4 \mathrm{~K}$ (green), and $6 \mathrm{~K}$ (red). The field is applied along the [110] (upper panel) and [001] (lower panel) directions. There is no hysteresis for $B \|[001]$ indicating no detectable spontaneous (zero-field) canting of the magnetic moments along the $c$ axis.

experimental $\mu$ values are higher than the curve described by Eq. (2) at least down to $2 \mathrm{~K}$. In order to obtain a better agreement with experimental results, we analyzed the data in a modified molecular field model expressed as [34]

$$
\frac{\mu}{\mu_{0}}=B_{S}\left(\frac{h}{T}+\frac{3 S}{S+1} \frac{T_{\mathrm{N}}\left[1+a\left(\mu / \mu_{0}\right)^{2}\right]}{T} \frac{\mu}{\mu_{0}}\right),
$$

where $h$ is a fictive magnetic field modeling the effect of short-range magnetic order above $T_{\mathrm{N}}$, and $a$ is a magnetoelastic parameter describing the magnetostrictive shift of $T_{\mathrm{N}}$ (Refs. [34,35]).

The fit using Eq. (3) for $\mathrm{HS} \mathrm{Co}^{2+}$ is shown by the solid line in the lower panel of Fig. 5. The latter approach yields a remarkably good account to the data with $h=0.06 \pm 0.01 \mathrm{~K}$ and $a=0.46 \pm 0.03$. These values are similar to those found for $\mathrm{Ba}_{2} \mathrm{CoGe}_{2} \mathrm{O}_{7}$ (Ref. [12]). A small but finite $h$ is responsible for the increase of $\mu$ above $T_{\mathrm{N}}$. We suggest that $h$ is due to the fluctuating short-range order persisting above $T_{\mathrm{N}}$, which was also observed for other layered antiferromagnets [36].

\section{Magnetization measurements}

An extremely weak canting (ferromagnetic component) derived from the neutron diffraction data agrees well with the tiny in-plane spontaneous magnetization observed in $\mathrm{Ca}_{2} \mathrm{CoSi}_{2} \mathrm{O}_{7}$ below $T_{\mathrm{N}} \approx 5.7 \mathrm{~K}$. Figure 6 presents the results of the bulk magnetization $(M)$ measurements just above $(6 \mathrm{~K})$ and below ( 2 and $4 \mathrm{~K}$ ) the Néel temperature with fields applied along the [110] and [001] directions. Taking into account the 


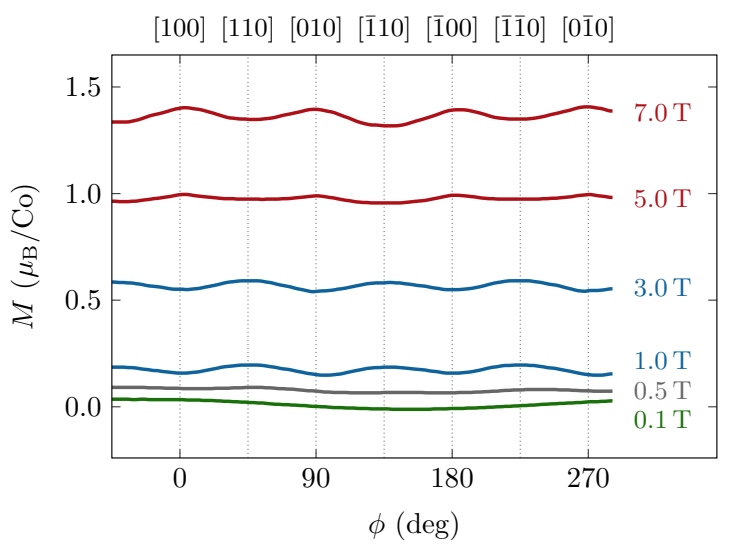

FIG. 7. Angular dependences of the magnetization $M$ at $2 \mathrm{~K} . \phi$ is the angle between the field and the [100] direction in the $a b$ plane. The corresponding crystallographic directions are indicated on the top of the figure.

magnitude of the Co magnetic moment we can estimate the mean value of zero field canting within the $a b$ plane to be less than $0.5^{\circ}$, which is too small to be reliably detected by unpolarised neutron diffraction. The canting along the [001] direction is not found even in the magnetization data (Fig. 6, lower panel).

The angular dependence of $M$ in the $a b$ plane was also investigated in fields up to $7 \mathrm{~T}$. Figure 7 shows the experimental results of the in-plane field-rotation measurements and the observed angular dependences at $T=2 \mathrm{~K}$. At low field $(0.1 \mathrm{~T})$ clear oscillations of $M$ with a $360^{\circ}$ period are found as expected for materials with weak spontaneous magnetization. At larger field $(0.5 \mathrm{~T})$ the period is approximately $180^{\circ}$ with tiny amplitude variation, indicating a weak orthorhombicity of the magnetic structure in agreement with field derivatives of the magnetization [13]. With further field increase the period changes to $90^{\circ}$ with a maximum magnetization at $\phi=45^{\circ}$ $(1-3 \mathrm{~T})$ or $\phi=0^{\circ}(5-7 \mathrm{~T})$.

This behavior can be explained within the magnetic structure determined here by neutron diffraction assuming the presence of magnetic domains (model $M 3 / M 4$ ). We note that the orthorhombicity in $\mathrm{Ca}_{2} \mathrm{CoSi}_{2} \mathrm{O}_{7}$ at low temperature is hidden due to the similar values of the $a$ and $b$ lattice parameters (equal within experimental precision) and the presence of almost equally populated $90^{\circ}$ twin domains (according to the structure refinement). If the applied field $B$ is parallel to one of the in-plane crystallographic axes $\langle 100\rangle /\langle 010\rangle$, then one type of the domains is characterized by $\mathrm{AF} \| B$ and its $90^{\circ}$ twins have $\mathrm{AF} \perp B$. With increasing field the canting inside the $\mathrm{AF} \perp B$ domains increases and their volumes increase at the expense of that of the AF $\| B$ domains. The latter ones try to keep their almost collinear configuration and, as a result, do not significantly contribute to the magnetization. In another case, when the field is applied along one of the $\langle 110\rangle$ directions, both types of magnetic domains have the same energy and thus equally contribute to the magnetization. As a result, at low fields the overall $\mathrm{F}$ component induced along the $\langle 100\rangle /\langle 010\rangle$ directions is smaller than that for $\langle 110\rangle$ (Fig. 6). However, at larger fields (above $4 \mathrm{~T}$ ), the situation is changed because those energetically unfavourable
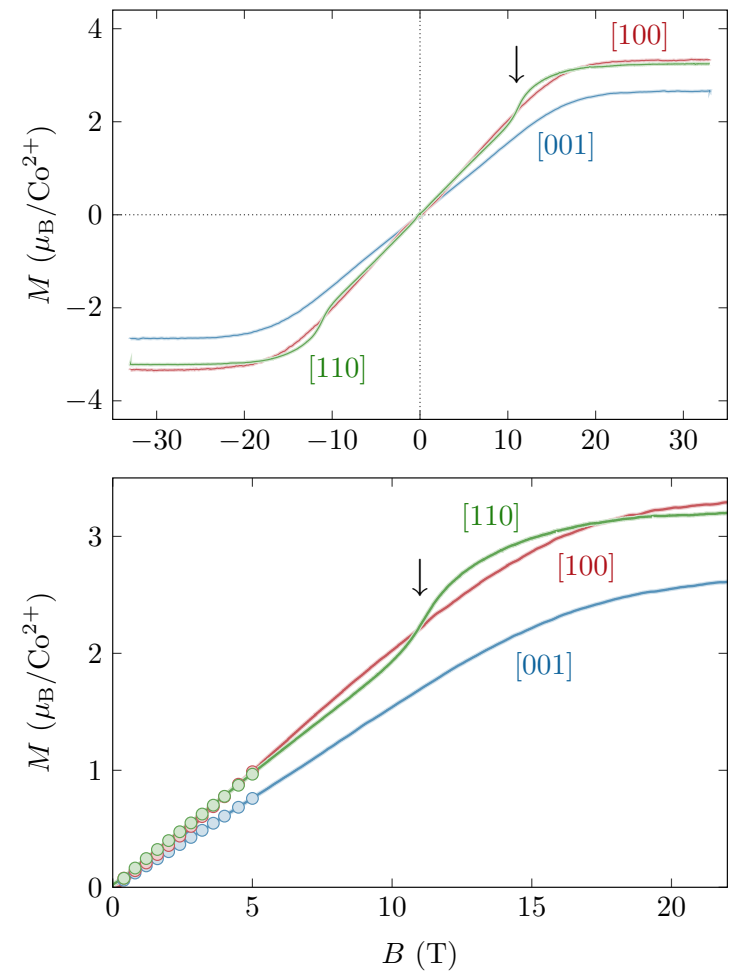

FIG. 8. Field dependences of the magnetization $M$ at $2 \mathrm{~K}$. Upper panel: the whole measured region. Lower panel: magnified selected part to emphasize the difference between the curves in the intermediate field range. The field is applied along the [100] (red), [110] (green), and [001] (blue) directions. Curves correspond to the measurements with a Bitter magnet, while symbols in the bottom panel represent the MPMS data. The magnetization anomaly at around $11 \mathrm{~T}$ for $\boldsymbol{B} \|$ [110], marked by an arrow, corresponds to the sign change of the ferroelectric polarization [13].

$90^{\circ}$ domains disappear. This identifies the $\langle 100\rangle /\langle 010\rangle$ axes as the easy axes of the antiferromagnetic order with the small ferromagnetic components being perpendicular to them.

Now, we turn to the analysis of the magnetization data in the intermediate- and high-field range, i.e., outside the region of the hysteresis. The magnetization data up to $32 \mathrm{~T}$ are shown in Fig. 8 for fields applied along the [100], [110], and [001] directions. The first important finding distinguishing $\mathrm{Ca}_{2} \mathrm{CoSi}_{2} \mathrm{O}_{7}$ from $\mathrm{Ba}_{2} \mathrm{CoGe}_{2} \mathrm{O}_{7}$ and $\mathrm{Sr}_{2} \mathrm{CoSi}_{2} \mathrm{O}_{7}$ is its seemingly smaller tetragonal anisotropy, as implied by susceptibility data in low magnetic fields. In all these compounds the magnetization is closely linear up to about $10 \mathrm{~T}$ irrespective of the direction of the applied fields [12]. However, the corresponding magnetic susceptibility components of $\mathrm{Ca}_{2} \mathrm{CoSi}_{2} \mathrm{O}_{7}$ measured in fields applied parallel and perpendicular to the [001] axis differ only by $\lesssim 20 \%$, while there is a factor of 2 difference in case of both $\mathrm{Ba}_{2} \mathrm{CoGe}_{2} \mathrm{O}_{7}$ and $\mathrm{Sr}_{2} \mathrm{CoSi}_{2} \mathrm{O}_{7}$. This alone implies a weaker tetragonal magnetic anisotropy in $\mathrm{Ca}_{2} \mathrm{CoSi}_{2} \mathrm{O}_{7}$. Another important difference is the magnetization anomaly observed at around $11 \mathrm{~T}$ (marked by an arrow in Fig. 8) for fields applied along the [110] direction but not for fields along the [100] axis. This indicates that the magnetic anisotropy within the (001) plane is stronger in $\mathrm{Ca}_{2} \mathrm{CoSi}_{2} \mathrm{O}_{7}$ compared to the other two compounds, which is in agreement with 
the stronger structural orthorhombicity found in $\mathrm{Ca}_{2} \mathrm{CoSi}_{2} \mathrm{O}_{7}$. This magnetization anomaly coincides with the sign change of the ferroelectric polarization, which happens in $\mathrm{Ca}_{2} \mathrm{CoSi}_{2} \mathrm{O}_{7}$ in a nearly discontinuous manner [13]. The instability of the magnetic state with zero electric polarization indicates the role of polarization-polarization coupling, which can be expressed also in terms of spin nematic or spin quadrupolar interactions. Indeed, the relevance of such fourth-order spin interactions has been predicted theoretically [37] and confirmed experimentally $[21,38]$ in the cases of $\mathrm{Ba}_{2} \mathrm{CoGe}_{2} \mathrm{O}_{7}$ and $\mathrm{Sr}_{2} \mathrm{CoGe}_{2} \mathrm{O}_{7}$. A third important difference is that while in $\mathrm{Ba}_{2} \mathrm{CoGe}_{2} \mathrm{O}_{7}$ and $\mathrm{Sr}_{2} \mathrm{CoSi}_{2} \mathrm{O}_{7}$ the magnetizations saturate nearly to the same value for each direction of the magnetic field $[4,12,13,39]$, for fields along the [001] axis in $\mathrm{Ca}_{2} \mathrm{CoSi}_{2} \mathrm{O}_{7}$ there is an intermediate-field magnetization plateau characterized by a considerably smaller value $\left(\sim 2.65 \mu_{\mathrm{B}}\right)$ than that observed for magnetic fields perpendicular to the [001] direction. The full saturation of the [001] axis magnetization was found to happen at much higher fields, namely at about $57 \mathrm{~T}$ (Ref. [13]). The large difference between the in-plane and outof-plane saturation fields together with the weak susceptibility anisotropy in low magnetic fields may imply that the tetragonal anisotropy in $\mathrm{Ca}_{2} \mathrm{CoSi}_{2} \mathrm{O}_{7}$ is not weak but has different anisotropy terms, such as the single-ion anostropy, exchange anisotropy and $g$-factor anisotropy, which compete and nearly compensate each other in the low-field magnetic state. These remarkable differences clearly speak for a different set of magnetic interactions realized in $\mathrm{Ca}_{2} \mathrm{CoSi}_{2} \mathrm{O}_{7}$ and underlie the importance of the current magnetic structure analysis.

\section{E. Crystal structure details at $2.5 \mathrm{~K}$}

In order to determine the structural parameters for $\mathrm{Ca}_{2} \mathrm{CoSi}_{2} \mathrm{O}_{7}$ at $2.5 \mathrm{~K}$, we performed a simultaneous refinement of crystal and magnetic structures from our neutron diffraction data using the $M 3 / M 4$ magnetic model selected in Sec. III B. The starting parameters were taken from the structure determined by neutron diffraction at $10 \mathrm{~K}$ (Ref. [17]). All atomic positions which are not restricted by symmetry were refined together with the isotropic atomic displacements $\left(U_{\text {iso }}\right)$, scale, extinction, and twin fraction parameters. The twin fraction represents the fractional volume of the crystal that the second domain type occupies. In order to reduce the large number of fitted parameters, caused by the low-temperature tripling of the unit cell, atoms of the same type were constrained to have identical $U_{\text {iso }}$ values. Besides this, no other constraints were used in the refinement process.

The agreement between the experimental and calculated data is shown in Fig. 3(f). Table II presents the refined atomic coordinates of the Co atoms. Full details of the refinement, including $U_{\text {iso }}$ parameters, bond lengths and angles, are deposited in the crystallographic information file (CIF) [40].

A comparison with the $10 \mathrm{~K}$ structure from neutron diffraction [17] shows negligible differences in the positional parameters with an average value of less than $1 \sigma$. As was already mentioned in Sec. III B, the twin fraction was found to be very close to a perfect twin value of 0.5 , which usually implies the existence of microtwins.
TABLE II. Fractional atomic coordinates $(x, y, z)$ for the commensurately modulated lock-in phase of the $(3 \times 3 \times 1)$ supercell refined in SG $P 2{ }_{1} 2{ }_{1} 2$ (twinned with respect to $m_{x y}$ ) according to the present single-crystal neutron diffraction data at $2.5 \mathrm{~K}$.

\begin{tabular}{lcccr}
\hline \hline Atom & WP & $x$ & $y$ & \multicolumn{1}{c}{$z$} \\
\hline $\mathrm{Co} 1$ & $2 e$ & 0 & 0 & $0.001(3)$ \\
$\mathrm{Co} 2$ & $4 c$ & $0.0066(4)$ & $0.3352(4)$ & $0.017(2)$ \\
$\mathrm{Co} 3$ & $4 c$ & $0.1628(4)$ & $0.1636(4)$ & $-0.006(3)$ \\
$\mathrm{Co} 4$ & $4 c$ & $0.3336(4)$ & $0.0018(4)$ & $0.015(2)$ \\
$\mathrm{Co} 5$ & $4 c$ & $0.3340(4)$ & $0.3313(4)$ & $0.017(2)$ \\
\hline \hline
\end{tabular}

\section{CONCLUSION}

The magnetic structure of $\mathrm{Ca}_{2} \mathrm{CoSi}_{2} \mathrm{O}_{7}$ at $2.5 \mathrm{~K}$ was solved based on the single-crystal neutron diffraction data and magnetic symmetry analysis. The results indicate the orthorhombic symmetry of the magnetic structure with MSG $P 2{ }_{1} 2_{1}^{\prime} 2^{\prime}$. The spin pattern shows a square-lattice in-plane $\mathrm{AF}$ order along the $a$ or $b$ axes for the $90^{\circ}$-type magnetic domains. Small canting (ferromagnetic component F) in the $a b$ plane perpendicular to the primary AF moment as well as out-of-plane along the $c$ axis is allowed by symmetry and leads to the existence of the tiny in-plane spontaneous magnetization below $T_{\mathrm{N}}$.

The magnetic structure model agrees with the angular dependence of the magnetization in the $a b$ plane. At zero magnetic field the magnitude of the averaged ordered magnetic moment of $\mathrm{Co}^{2+}$ ions is found to be about $2.8 \mu_{\mathrm{B}}$ according to the neutron diffraction data. The high-field saturation value from bulk magnetization measurements is noticeably higher, exceeding $3.3 \mu_{\mathrm{B}}$, probably due to the spin gap induced by single ion anisotropy similar to $\mathrm{Ba}_{2} \mathrm{CoGe}_{2} \mathrm{O}_{7}$.

We also report here the structural parameters of $\mathrm{Ca}_{2} \mathrm{CoSi}_{2} \mathrm{O}_{7}$ in the low-temperature magnetoelectric state. No evidence for a structural phase transition upon the magnetic phase transition at $5.7 \mathrm{~K}$ was observed by neutron diffraction, and the crystal structure at $2.5 \mathrm{~K}$ is found to correspond well to that at $10 \mathrm{~K}$.

Our neutron scattering experiments as well as bulk magnetization studies indicate pronounced differences between the magnetic state of $\mathrm{Ca}_{2} \mathrm{CoSi}_{2} \mathrm{O}_{7}$ if compared to other magnetically ordered mellilites such as $\mathrm{Ba}_{2} \mathrm{CoGe}_{2} \mathrm{O}_{7}$ and $\mathrm{Sr}_{2} \mathrm{CoSi}_{2} \mathrm{O}_{7}$. The structural parameters reported here (both for the nuclear and magnetic order) of $\mathrm{Ca}_{2} \mathrm{CoSi}_{2} \mathrm{O}_{7}$ can serve as a profound experimental basis to develop microscopic models describing the multiferroic nature and the peculiar magnetoelectric phenomena in melilites. Neutron diffraction measurements in the intermediate field region, where a magnetization plateau is observed for fields along the [001] axis, would provide further insight into the nature of the magnetic phases of $\mathrm{Ca}_{2} \mathrm{CoSi}_{2} \mathrm{O}_{7}$.

\section{ACKNOWLEDGMENTS}

This work was partly supported by the BMBF under Contract No. 05K13PA3, by the Hungarian Research Funds OTKA K 108918 and by the UNKP-16-3/III New National Excellence Program of the Ministry of Human Capacities. This project has also received funding from the European Union's Seventh 
Framework Program for research, technological development and demonstration under the NMI3-II Grant No. 283883 and by the Swiss National Science Foundation. We acknowledge the support of the HFML-RU/FOM, member of the European
Magnetic Field Laboratory (EMFL). Part of the work is based upon experiments performed at the HEiDi instrument which is operated by RWTH Aachen University and Forschungszentrum Jülich GmbH (Jülich Aachen Research Alliance JARA).
[1] M. Akaki, J. Tozawa, D. Akahoshi, and H. Kuwahara, J. Phys.: Conf. Ser. 150, 042001 (2009).

[2] M. Akaki, J. Tozawa, M. Hitomi, D. Akahoshi, and H. Kuwahara, J. Phys.: Conf. Ser. 200, 012003 (2010).

[3] I. Kézsmárki, D. Szaller, S. Bordács, V. Kocsis, Y. Tokunaga, Y. Taguchi, H. Murakawa, Y. Tokura, H. Engelkamp, T. Rõõm, and U. Nagel, Nat. Commun. 5, 3203 (2014).

[4] M. Akaki, J. Tozawa, D. Akahoshi, and H. Kuwahara, Appl. Phys. Lett. 94, 212904 (2009).

[5] H. Murakawa, Y. Onose, S. Miyahara, N. Furukawa, and Y. Tokura, Phys. Rev. Lett. 105, 137202 (2010).

[6] J. W. Kim, S. Khim, S. H. Chun, Y. Jo, L. Balicas, H. T. Yi, S.-W. Cheong, N. Harrison, C. D. Batista, J. H. Han, and K. H. Kim, Nat. Commun. 5, 4419 (2014).

[7] S. Bordacs, I. Kezsmarki, D. Szaller, L. Demko, N. Kida, H. Murakawa, Y. Onose, R. Shimano, T. Room, U. Nagel, S. Miyahara, N. Furukawa, and Y. Tokura, Nat. Phys. 8, 734 (2012).

[8] I. Kézsmárki, N. Kida, H. Murakawa, S. Bordács, Y. Onose, and Y. Tokura, Phys. Rev. Lett. 106, 057403 (2011).

[9] J. M. Perez-Mato and J. L. Ribeiro, Acta Crystallogr. A 67, 264 (2011).

[10] D. Szaller, S. Bordács, V. Kocsis, T. Rõõm, U. Nagel, and I. Kézsmárki, Phys. Rev. B 89, 184419 (2014).

[11] A. Sazonov, M. Meven, G. Roth, R. Georgii, I. Kézsmárki, V. Kocsis, Y. Tokunaga, Y. Taguchi, Y. Tokura, and V. Hutanu, J. Appl. Crystallogr. 49, 556 (2016).

[12] V. Hutanu, A. P. Sazonov, M. Meven, G. Roth, A. Gukasov, H. Murakawa, Y. Tokura, D. Szaller, S. Bordács, I. Kézsmárki, V. K. Guduru, L. C. J. M. Peters, U. Zeitler, J. Romhányi, and B. Náfrádi, Phys. Rev. B 89, 064403 (2014).

[13] M. Akaki, H. Kuwahara, A. Matsuo, K. Kindo, and M. Tokunaga, J. Phys. Soc. Jpn. 83, 093704 (2014).

[14] K. Kusaka, K. Hagiya, M. Ohmasa, Y. Okano, M. Mukai, K. Iishi, and N. Haga, Phys. Chem. Miner. 28, 150 (2001).

[15] K. Hagiya, K. Kusaka, M. Ohmasa, and K. Iishi, Acta Crystallogr. B 57, 271 (2001).

[16] H. Böhm, M. Riester, and V. Petricek, Z. Kristallogr. 215, 102 (2000).

[17] A. Sazonov, V. Hutanu, M. Meven, G. Roth, I. Kézsmárki, H. Murakawa, Y. Tokura, and B. Náfrádi, Acta Crystallogr. B 72, 126 (2016)

[18] M. Riester and H. Böhm, Z. Kristallogr. 212, 506 (1997).

[19] Z. H. Jia, A. K. Schaper, W. Massa, W. Treutmann, and H. Rager, Acta Crystallogr. B 62, 547 (2006).

[20] K. Penc, J. Romhányi, T. Rõõm, U. Nagel, A. Antal, T. Fehér, A. Jánossy, H. Engelkamp, H. Murakawa, Y. Tokura, D. Szaller,
S. Bordács, and I. Kézsmárki, Phys. Rev. Lett. 108, 257203 (2012).

[21] M. Soda, M. Matsumoto, M. Månsson, S. Ohira-Kawamura, K. Nakajima, R. Shiina, and T. Masuda, Phys. Rev. Lett. 112, 127205 (2014).

[22] M. Meven and A. Sazonov, J. Large-scale Res. Facilities 1, A7 (2015).

[23] A. P. Sazonov, Davinci: Software for visualization and processing of single-crystal diffraction data measured with a point detector, 2015, http://davinci.sazonov.org.

[24] M. S. Lehmann and F. K. Larsen, Acta Crystallogr. A 30, 580 (1974).

[25] V. Petříček, M. Dušek, and L. Palatinus, Z. Kristallogr. 229, 345 (2014).

[26] J. M. Perez-Mato, S. V. Gallego, E. S. Tasci, L. Elcoro, G de la Flor, and M. I. Aroyo, Annu. Rev. Mater. Res. 45, 217 (2015).

[27] T. Chatterji, Neutron Scattering from Magnetic Materials (Elsevier, Amsterdam, 2006), p. 572.

[28] E. E. Rodriguez, C. Stock, K. L. Krycka, C. F. Majkrzak, P. Zajdel, K. Kirshenbaum, N. P. Butch, S. R. Saha, J. Paglione, and M. A. Green, Phys. Rev. B 83, 134438 (2011).

[29] M. Greven, R. J. Birgeneau, Y. Endoh, M. A. Kastner, B. Keimer, M. Matsuda, G. Shirane, and T. R. Thurston, Phys. Rev. Lett. 72, 1096 (1994).

[30] S. T. Bramwell and P. C. W. Holdsworth, Phys. Rev. B 49, 8811 (1994).

[31] M. Greven, R. J. Birgeneau, Y. Endoh, M. A. Kastner, M. Matsuda, and G. Shirane, Z. Phys. B 96, 465 (1995).

[32] M. Renninger, Z. Phys. 106, 141 (1937).

[33] E. Rossmanith, Acta Crystallogr. A 62, 174 (2006).

[34] A. B. Beznosov, B. I. Belevtsev, E. L. Fertman, V. A. Desnenko, D. G. Naugle, K. D. D. Rathnayaka, and A. Parasiris, Low Temp. Phys. 28, 556 (2002).

[35] A. B. Beznosov, E. L. Fertman, V. V. Eremenko, P. P. Pal-Val, V. P. Popov, and N. N. Chebotayev, Low Temp. Phys. 27, 320 (2001).

[36] A. Antal, T. Fehér, B. Náfrádi, L. Forró, and A. Jánossy, Phys. Status Solidi B 249, 1004 (2012).

[37] J. Romhányi, M. Lajkó, and K. Penc, Phys. Rev. B 84, 224419 (2011).

[38] M. Akaki, D. Yoshizawa, A. Okutani, T. Kida, J. Romhányi, K. Penc, and M. Hagiwara, arXiv:1702.04158.

[39] H. Murakawa, Y. Onose, S. Miyahara, N. Furukawa, and Y. Tokura, Phys. Rev. B 85, 174106 (2012).

[40] ICSD, CSD-No. 432983, http://www.fiz-karlsruhe.de. 